\title{
LIOUVILLIAN INTEGRABILITY VERSUS DARBOUX POLYNOMIALS
}

\author{
JAUME LLIBRE ${ }^{1}$, CLAUDIA VALLS ${ }^{2}$ AND XIANG ZHANG ${ }^{3}$ \\ ABSTRACT. In this note we establish some relations among Liou- \\ villian integrable polynomial differential systems, their degree and \\ the existence of Darboux polynomials. As an application of our \\ main results we prove that the Liénard polynomial differential sys- \\ tem $\dot{x}=y, \dot{y}=-f(x) y-g(x)$ with $\operatorname{deg} f>\operatorname{deg} g$ is not Liouvillian \\ integrable.
}

\section{Introduction AND STATEMENT OF the MAIN RESUlts}

One of the classical problems in the dynamical systems is to determine when a system has first integrals. The existence of first integrals together with other invariants like constants of motion, conserved quantities, integrating factors, Jacobian multipliers and Lie symmetries, provide different techniques for studying the dynamics of differential equations coming from different topics such as celestial mechanics, physics, engineering, biology and so on. But in this note we put our attention on the Liouvillian integrability and its related invariants.

Consider polynomial differential systems

$$
\dot{x}=P(x), \quad x \in \mathbb{C}^{n},
$$

where the dot denotes derivative with respect to the independent variable $t$, and $P(x)=\left(P_{1}(x), \ldots, P_{n}(x)\right)$ is an $n$ dimensional vector valued polynomial. For polynomial differential systems $(1)$ there are three related notions: Darboux polynomial, Darboux integrability and Liouvillian integrability. In this note we will present some relations among these three notions.

A function $H(x)$ is a first integral of system (1) if it is continuous and defined in $\mathbb{C}^{n}$ except perhaps in a zero Lebesgue measure subset $\Omega_{1}$, it is not locally constant on any positive Lebesgue measure subset of $\mathbb{C}^{n} \backslash \Omega_{1}$, and $H(x)$ is constant along each orbit of system (1) in $\mathbb{C}^{n} \backslash \Omega_{1}$.

2010 Mathematics Subject Classification. 34A34; 34C20; 34C14.

Key words and phrases. Polynomial differential system; Liouvillian integrability; Darboux Jacobian multiplier; Darboux polynomial. 
A function $H(x)$ is a Darboux first integral of system (1) if it is a first integral and is a function of Darboux type, i.e. it is of the form

$$
f_{1}^{r_{1}}(x) \ldots f_{k}^{r_{k}}(x) e^{h(x) / g(x)},
$$

where $r_{j} \in \mathbb{C}, f_{j}, g, h$ are polynomials for $j=1, \ldots, k$, and $g$ and $h$ are coprime.

A function $H(x)$ is a Liouvillian first integral of system (1) if it is a first integral and belongs to a Liouvillian field extension of $\mathbb{C}(x)$. As usual $\mathbb{C}(x)$ denotes the field of all rational functions in the variable $x \in$ $\mathbb{C}^{n}$. Let $\Delta$ be a given set of commuting derivations in the differentiable field $\mathbb{C}(x)$.

Recall that a differential field extension $L$ of $\mathbb{C}(x)$ is Liouvillian if this extension can be written as a tower of the form

$$
K_{0}=\mathbb{C}(x) \subset K_{1} \subset \ldots \subset K_{l}=L,
$$

such that each field extension satisfies one of the following three ones:

(a) $K_{i+1}$ is a finite algebraic extension of $K_{i}$; or

(b) $K_{i+1}=K_{i}(t)$, where $t$ satisfies that for each $\delta \in \Delta$, $\frac{\delta t}{t} \in K_{i}$; or

(c) $K_{i+1}=K_{i}(t)$, where $t$ satisfies that for each $\delta \in \Delta, \delta t \in K_{i}$.

System (1) is Liouvillian integrable, if it has $n-1$ functionally independent Liouvillian first integrals in $\mathbb{C}^{n}$. Recall that $k$ functions $H_{1}(x), \ldots, H_{k}(x)$ are functionally independent in $\mathbb{C}^{n}$ if their gradients $\nabla H_{1}, \ldots, \nabla H_{k}$ have rank $k$ in $\mathbb{C}^{n}$ except perhaps in a zero Lebesgue measure subset. System (1) is Darboux integrable, if it has $n-1$ functionally independent Darboux first integrals in $\mathbb{C}^{n}$.

Here $\mathbb{C}[x]$ denotes the ring of all polynomials in the variable $x \in \mathbb{C}^{n}$. A polynomial $f(x) \in \mathbb{C}[x] \backslash \mathbb{C}$ is a Darboux polynomial of the polynomial differential system (1) if there exists a $k(x) \in \mathbb{C}[x]$ such that

$$
\mathcal{X}(f)(x)=k(x) f(x), \quad x \in \mathbb{C}^{n},
$$

where $\mathcal{X}$ is the vector field associated to system (1), i.e.

$$
\mathcal{X}=P_{1}(x) \frac{\partial}{\partial x_{1}}+\ldots+P_{n}(x) \frac{\partial}{\partial x_{n}} .
$$

A $C^{1}$ non-zero function $J$ is a Jacobian multiplier of system (1) if it is defined in $\mathbb{C}^{n}$ except perhaps in a zero Lebesgue measure subset $\Omega_{1}$, and satisfies

$$
\operatorname{div}(J f) \equiv 0, \quad \text { i.e., } \quad \mathcal{X}(J)=-J \operatorname{div} \mathcal{X}, \quad x \in \mathbb{C}^{n} \backslash \Omega_{1},
$$

where $\operatorname{div} \mathcal{X}$ denotes the divergence of the vector field $\mathcal{X}$. A Jacobian multiplier is Darboux if it is of Darboux type. As convention, if system 
(1) is two dimensional, a Jacobian multiplier is called an integrating factor.

Darboux theory of integrability was started in 1878 by Darboux $[7,8]$, who provided a method to construct first integral using Darboux polynomials. This theory has been greatly developed in different aspects, see for instance $[2,5,6,10,11,13,22,28,25,26,27,39]$ and the references therein. The Darboux theory of integrability has also been broadly applied to study dynamics of differential systems, for instance the center problem, limit cycles and global dynamical analysis, see for example $[5,9,17,18,31,35,36,37]$ and the references quoted there.

Singer [30] in 1992 proved the equivalence between Liouvillian integrability and Darboux integrability of planar polynomial differential systems, see also [4]. Zhang [38] proved that Liouvillian integrability of higher dimensional polynomial differential systems implies the existence of Darboux Jacobian multipliers. In general Darboux integrability depends on the existence of Darboux polynomials and exponential factors. There are plenty of results studying the existence of Darboux polynomials, see for example $[1,14,23,24,32,33,34,40]$ and the references mentioned there.

Our next result shows that a Liouvillian integrable polynomial differential system with conditions on the degree of the system implies the existence of Darboux polynomials, but this will not be always the case as we shall see in Proposition 3.

Theorem 1. Let $k_{j}$ be the degree of the polynomial $P_{j}\left(x_{1}, \ldots, x_{n}\right)$ with respect to the variable $x_{k}$ for some $k \in\{1, \ldots, n\}$. If the polynomial differential system (1) has a Darboux Jacobian multiplier and $k_{\ell}>$ $\max \left\{k_{1}, \ldots, \hat{k}_{\ell}, \ldots, k_{n}\right\}+1$ for some $\ell \in\{1, \ldots, n\}$, then system (1) has a Darboux polynomial, where $\hat{k}_{\ell}$ denotes the absence of $k_{\ell}$.

Combining Theorem 1.2 of [38], we can get the next result.

Corollary 2. If the polynomial differential system (1) is Liouvillian integrable and $k_{\ell}>\max \left\{k_{1}, \ldots, \hat{k}_{\ell}, \ldots, k_{n}\right\}+1$ for some $\ell \in\{1, \ldots, n\}$, then system (1) has a Darboux polynomial, where we have used the notation given in Theorem 1.

We remark that Theorem 1 and Corollary 2 are extension of Theorem 1 and Corollary 2 of [12] from two dimensional polynomial differential systems to any finite dimensional polynomial differential systems.

Our next result shows that Theorem 1 and Corollary 2 in general do not hold if $k_{\ell} \leq \max \left\{k_{1}, \ldots, \hat{k}_{\ell}, \ldots, k_{n}\right\}+1$ for all $\ell \in\{1, \ldots, n\}$. 
Proposition 3. If $k_{\ell}>\max \left\{k_{1}, \ldots, \hat{k}_{\ell}, \ldots, k_{n}\right\}+1$ does not hold for all $\ell=1, \ldots, n$, then the following statements hold.

(a) There exist examples of system (1) which are Liouvillian integrable and have no Darboux polynomials.

(b) There also exist examples of system (1) which are Liouvillian integrable and have Darboux polynomials.

If systems (1) satisfy the degree condition $k_{\ell}>\max \left\{k_{1}, \ldots, \hat{k}_{\ell}, \ldots, k_{n}\right\}+$ 1 , have a Darboux polynomial and are integrable, then they may not be Liouvillian integrable as the following proposition shows.

Proposition 4. There exist integrable systems (1) such that $k_{\ell}>$ $\max \left\{k_{1}, \ldots, \hat{k}_{\ell}, \ldots, k_{n}\right\}+1$, having a Darboux polynomial and without Liouvillian first integrals.

As an application of Theorem 1 and Corollary 2 to Liénard differential system, we get the following result.

Theorem 5. The polynomial Liénard differential systems

$$
\dot{x}=y, \quad \dot{y}=-f(x) y-g(x),
$$

with $f$ and $g$ polynomials in $x$, are not Liouvillian integrable provided that $\operatorname{deg} f>\operatorname{deg} g$ and $g(x) \not \equiv 0$.

About the Liouvillian integrability of Liénard polynomial differential systems, Llibre and Valls [19] characterized the Liouvillian integrability of the Liénard polynomial differential system $\dot{x}=y, \dot{y}=-c x-f(x) y$. In [20] the authors characterized the Liouvillian integrability of the Liénard differential system (3) with $\operatorname{deg} f \geq \operatorname{deg} g \geq 2$. Recently Llibre and Valls [21] proved that system (3) is not Liouvillian integrable provided that $\operatorname{deg} g=\operatorname{deg} f+1$. We note that Theorem 5 can allow $\operatorname{deg} g=0$, whereas $\operatorname{deg} g>0$ in [19] and [20]. Here we provide a different and easy proof than $[19,20]$ on non-Liouvillian integrability for the class of Liénard differential systems studied in Theorem 5.

The rest of this paper is devoted to prove our main results.

\section{Proof of the MAIN RESUlts}

For proving our theorems we need the following result, which is well known for the mathematicians working in the Darboux theory of integrability, but as far as we know there is not published a proof.

Proposition 6. If a Darboux function

$$
F=f_{1}^{r_{1}} \ldots f_{m}^{r_{m}} \exp \left(\frac{h}{g}\right)
$$


with $g$ and $h$ coprime and the $f_{j}$ 's irreducible, is a Jacobian multiplier of system (1), then all $g$ and $f_{j}$ for $j=1, \ldots, m$ are Darboux polynomials of system (1).

Proof. Recall that $\mathcal{X}$ is the vector field associated to system (1). By assumption $F$ is a Jacobian multiplier, so we have $\mathcal{X}(F)=-F \operatorname{div} \mathcal{X}$. Writing this equality in components we obtain

$$
\begin{aligned}
& g^{2} \sum_{j=1}^{m} r_{j} f_{1}^{r_{1}} \ldots f_{j-1}^{r_{j-1}} f_{j}^{r_{j}-1} f_{j+1}^{r_{j+1}} \ldots f_{m}^{r_{m}} \mathcal{X}\left(f_{j}\right) \\
& \quad+f_{1}^{r_{1}} \ldots f_{m}^{r_{m}}(\mathcal{X}(h) g-h \mathcal{X}(g))=-f_{1}^{r_{1}} \ldots f_{m}^{r_{m}} g^{2} \operatorname{div} \mathcal{X} .
\end{aligned}
$$

From this equality we get that for any $j \in\{1, \ldots, m\}$ the polynomial $f_{j}$ divides $g^{2} \mathcal{X}\left(f_{j}\right)$. This implies that $f_{j}$ divides $g$ or $\mathcal{X}\left(f_{j}\right)$. If the latter happens, there exists a $k_{j}(x) \in \mathbb{C}[x]$ such that $\mathcal{X}\left(f_{j}\right)=k_{j}(x) f_{j}(x)$, and consequently $f_{j}$ is a Darboux polynomial. If the former happens, we can write $g(x)=g_{0}(x) f_{j}^{l}(x)$, where $g_{0}$ is a polynomial relative prime with $f_{j}$, and $l$ is a positive integer. Equating the power of $f_{j}$ in the components of (4) we get that $f_{j}^{l}$ divides $h \mathcal{X}(g)$. Since $g$ and $h$ are relatively prime and $f_{j}$ is a factor of $g$, we must have $f_{j}^{l}$ divides $\mathcal{X}(g)$. In addition $\mathcal{X}(g)=\mathcal{X}\left(g_{0}\right) f_{j}^{l}+l g_{0} f_{j}^{l-1} \mathcal{X}\left(f_{j}\right)$. This shows that $f_{j}$ divides $\mathcal{X}\left(f_{j}\right)$. So we have proved that all $f_{j}$ 's are Darboux polynomials of system (1).

Since each $f_{j}$ is a Darboux polynomial, we can erase the factor $f_{1}^{r_{1}} \ldots f_{m}^{r_{m}}$ from equation (4). Then we get from this resulting equation that $g$ divides $h \mathcal{X}(g)$. Consequently $g$ divides $\mathcal{X}(g)$ because $g$ and $h$ are relatively prime. This shows that $g$ is a Darboux polynomial. This completes the proof of the proposition.

Proof of Theorem 1. Without loss of generality we prove the theorem for $\ell=n$. Set $\bar{x}=\left(x_{1}, \ldots, x_{n-1}\right)$, and write system (1) in the form

$$
\dot{x}_{j}=p_{0}^{(j)}(\bar{x})+p_{1}^{(j)}(\bar{x}) x_{n}+\ldots+p_{k_{j}}^{(j)}(\bar{x}) x_{n}^{k_{j}}, \quad j=1, \ldots, n
$$

where the $p_{i}^{(j)}(x)$ 's are polynomials for $j=1, \ldots, n$ and $i=0,1, \ldots, k_{j}$, and $p_{k_{n}}^{(n)} \not \equiv 0$.

By assumption system (1) has a Darboux Jacobian multiplier, denoted by $J$. Recall that a Darboux Jacobian multiplier is a Jacobian multiplier of the form (2). From Proposition 6, if system (5) has no Darboux polynomials then the Darboux Jacobian multiplier $J$ must be of the form $J=e^{h(x)}$ with $h(x)$ a polynomial. Set $h(x)=$ $h_{0}(\bar{x})+h_{1}(\bar{x}) x_{1}+\ldots+h_{r}(\bar{x}) x_{n}^{r}$ with $r \geq 0$ and $h_{r}(\bar{x}) \not \equiv 0$. 
By definition of Jacobian multiplier we get that

$$
\sum_{j=1}^{n-1}\left(\sum_{s=0}^{k_{j}} p_{s}^{(j)}(\bar{x}) x_{n}^{s}\right) \sum_{i=0}^{r} \frac{\partial h_{i}(\bar{x})}{\partial x_{j}} x_{n}^{i}+\sum_{s=0}^{k_{n}} p_{s}^{(n)}(\bar{x}) x_{n}^{s} \sum_{i=0}^{r} i h_{i}(\bar{x}) x_{n}^{i-1}
$$

(6)

$$
=-\left(\sum_{i=0}^{r} h_{i}(\bar{x}) x_{n}^{i}\right)\left(\sum_{j=1}^{n-1} \sum_{s=0}^{k_{j}} \frac{\partial p_{s}^{(j)}(\bar{x})}{\partial x_{j}} x_{n}^{s}+\sum_{s=0}^{k_{n}} s p_{s}^{(n)}(\bar{x}) x_{n}^{s-1}\right) .
$$

By equating the coefficients of $x^{k_{n}+r-1}$, we get from assumption $k_{n}>$ $\max \left\{k_{1}, \ldots, k_{n-1}\right\}+1$ that $\left(r+k_{n}\right) p_{k_{n}}^{(n)}(\bar{x}) h_{r}(\bar{x})=0$. This is a contradiction because $r \geq 0, k_{n}>0$, and $p_{k_{n}}^{(n)}(\bar{x}) h_{r}(\bar{x}) \neq 0$. This completes the proof of the theorem.

The next result was proved in [38].

Theorem 7. If system (1) is Liouvillian integrable, then it has a Darboux Jacobian multiplier.

Proof of Corollary 2. Its proof follows immediately from Theorems 1 and 7.

Proof of Proposition 3. We first prove statement (a). Consider the following polynomial differential system in $\mathbb{R}^{3}$

$$
\dot{x}=2 x z-z^{k}, \quad \dot{y}=3 y z-z^{\ell}, \quad \dot{z}=-1,
$$

with $k, \ell>1$ even. Clearly this system satisfies the degree condition of the proposition. We can check that system (7) has the first integrals

(8) $H_{1}(x, y, z)=x e^{z^{2}}-\int z^{k} e^{z^{2}} d z, H_{2}(x, y, z)=y e^{\frac{3}{2} z^{2}}-\int z^{\ell} e^{\frac{3}{2} z^{2}} d z$.

Clearly they are functionally independent. Moreover $H_{1}$ and $H_{2}$ are Liouvillian because they are obtained by taking exponential and integrating from polynomial functions, and each of these steps belongs to the tower elements in the Liouvillian field extension of $\mathbb{C}(x)$.

Next we prove that system (7) has no Darboux polynomials. We should mention that we cannot directly use the two first integrals $H_{1}$ and $H_{2}$ to prove our arguments, even through any other first integral is functionally dependent of $H_{1}$ and $H_{2}$, because we do not know if system (7) has other first integrals, whose level surfaces contain invariant algebraic surfaces.

Let $\mathcal{Y}$ be the vector field associated to system (7). If $F(x, y, z)$ is a Darboux polynomial, then there exists a polynomial $K(x, y, z)$ such 
that $\mathcal{Y}(F)=K F$. Write $F$ and $K$ as polynomials in the variable $x$, i.e.

$$
\begin{aligned}
& F(x, y, z)=f_{0}(y, z)+f_{1}(y, z) x+\ldots+f_{m}(y, z) x^{m} \\
& K(x, y, z)=k_{0}(y, z)+k_{1}(y, z) x+\ldots+k_{r}(y, z) x^{r}
\end{aligned}
$$

with $m, r \geq 0$ integers, and the coefficients polynomials in $y$ and $z$. Then we get from $\mathcal{Y}(F)=K F$ that

$$
\left(2 x z-z^{k}\right) \sum_{j=0}^{m} j f_{j}(y, z) x^{j-1}+\left(3 y z-z^{\ell}\right) \sum_{j=0}^{m} \partial_{y} f_{j}(y, z) x^{j}
$$

$$
-\sum_{j=0}^{m} \partial_{z} f_{j}(y, z) x^{j}=\left(\sum_{j=0}^{r} k_{j}(y, z) x^{j}\right)\left(\sum_{j=0}^{m} f_{j}(y, z) x^{j}\right) .
$$

Without loss of generality we can assume that $f_{m}(y, z) \not \equiv 0$. Equating the coefficients of $x^{k}$ in (9) with $k>m$ we get that $k_{1}(y, z)=\ldots=$ $k_{r}(y, z) \equiv 0$. Equating the coefficients of $x^{m}$ in (9) gives

$$
\left(3 y z-z^{\ell}\right) \partial_{y} f_{m}(y, z)-\partial_{z} f_{m}(y, z)=\left(k_{0}(y, z)-2 m z\right) f_{m}(y, z) .
$$

For the coefficients of $x^{s}$ in (9), $s=0,1, \ldots, m-1$, we have

$$
\begin{aligned}
& \left(3 y z-z^{\ell}\right) \partial_{y} f_{s}(y, z)-\partial_{z} f_{s}(y, z) \\
& \quad=\left(k_{0}(y, z)-2 s z\right) f_{s}(y, z)+(s+1) z^{k} f_{s+1}(y, z) .
\end{aligned}
$$

If $f_{m} \neq$ constant we set

$$
f_{m}(y, z)=g_{0}(z)+g_{1}(z) y+\ldots+g_{q}(z) y^{q},
$$

with $q$ a nonnegative integer and the $g_{j}$ 's polynomials in $z$ for $j=$ $0,1, \ldots, q$ such that $g_{q}(z) \not \equiv 0$. Substituting the expression of $f_{m}$ into equation (10) and equating the coefficients of $y^{j}$ for $j \geq q$, we get that $k_{0}(y, z)=k_{0}(z)$ and

$$
g_{q}^{\prime}(z)=-\left(k_{0}(z)-2 m z-3 q z\right) g_{q}(z) .
$$

Its general solution is

$$
g_{q}(z)=C e^{\int\left(2 m z+3 q z-k_{0}(z)\right) d z},
$$

where $C$ is a constant. So this linear equation has a polynomial solution only in the case $k_{0}(z)=(2 m+3 q) z$, and $g_{q}(z)=C$ is a constant. Consequently $q>0$ because by assumptions $f_{m}(y, z)$ is not a constant. Equating the coefficient of $y^{q-1}$ in equation (10) we get

$$
g_{q-1}^{\prime}(z)=-3 z g_{q-1}-q C z^{\ell} .
$$


This linear equation has the general solution

$$
g_{q-1}(z)=e^{-\frac{3}{2} z^{2}}\left(c-q C \int z^{\ell} e^{\frac{3}{2} z^{2}} d z\right),
$$

where $c$ is the integrating constant. Since $\ell$ is even, we set $\ell=2 m$, then we have

$$
\begin{array}{r}
\int z^{2 m} e^{\frac{3}{2} z^{2}} d z=\frac{e^{\frac{3}{2} z^{2}}}{3^{m}}\left(\sum_{i=0}^{m-1}(-1)^{i} 3^{m-i-1} \frac{(2 m-1) ! !}{(2 m-2 i-1) ! !} z^{2 m-2 i-1}\right. \\
\left.+(-1)^{m}(2 m-1) ! ! \int e^{\frac{3}{2} z^{2}} d z\right),
\end{array}
$$

where $(2 m+1) ! !=(2 m+1)(2 m-1) \ldots 3 \cdot 1$. In order that $g_{q-1}(z)$ be a polynomial, we must have $c=0$ and $q C=0$, a contradiction because neither $q$ nor $C$ can be zero.

If $f_{m}(y, z)=K$ equal to a non-zero constant, then $m>0$, otherwise $F(x, y, z)$ is a constant. We get from $(10)$ that $k_{0}(z)=2 m z$. Now equation (11) for $s=m-1$ is

$$
\left(3 y z-z^{\ell}\right) \partial_{y} f_{m-1}(y, z)-\partial_{z} f_{m-1}(y, z)=2 z f_{m-1}(y, z)+m K z^{k}
$$

Since $k>1$ the solution of this last equation cannot be a constant. Set

$$
f_{m-1}(y, z)=h_{0}(z)+h_{1}(z) y+\ldots+h_{p}(z) y^{p}, \quad p \geq 0 .
$$

Then we get from equation (12) that

$$
h_{p}^{\prime}(z)=(3 p-2) z h_{p}(z) .
$$

The general solution of equation (13) is $h_{p}(z)=c \exp \left(\frac{3 p-2}{2} z^{2}\right)$ where $c$ is a constant. Since $p$ is a nonnegative integer, it forces that $h_{p}$ must be either zero or not a polynomial. This implies that $f_{m-1}(y, z)$ is not a polynomial. This contradiction proves statement $(a)$.

Now we shall prove statement $(b)$. Consider the polynomial differential system in $\mathbb{R}^{3}$

$$
\dot{x}=2 x z-z^{k}, \quad \dot{y}=2 y z-z^{\ell}, \quad \dot{z}=-1 .
$$

For any positive integers $k$ and $l$, system (14) has at least one Darboux polynomial. Indeed, since system (14) has the functionally independent Liouvillian first integrals

$$
H_{1}(x, y, z)=x e^{z^{2}}-\int z^{k} e^{z^{2}} d z, H_{2}(x, y, z)=y e^{z^{2}}-\int z^{\ell} e^{z^{2}} d z
$$


If one of $k$ and $\ell$ is odd, for illustration we assume that $k=2 m+1$. Some calculations show that

$$
H_{1}(x, y, z)=e^{z^{2}}\left(x-\frac{1}{2} \sum_{j=0}^{m}(-1)^{j} \frac{m !}{(m-j) !} z^{2(m-j)}\right) .
$$

Since $H_{1}(x, y, z)=0$ is an invariant set, so

$$
F(x, y, z):=x-\frac{1}{2} \sum_{j=0}^{m}(-1)^{j} \frac{m !}{(m-j) !} z^{2(m-j)}=0,
$$

is an invariant surface. This proves that $F(x, y, z)$ is a Darboux polynomial of system (14).

If $k$ and $\ell$ are both even, set $k=2 p$ and $\ell=2 q$. Since for $m \in\{p, q\}$

$$
\begin{array}{r}
\int z^{2 m} e^{z^{2}} d z=\frac{1}{2} e^{z^{2}}\left(\sum_{i=0}^{m-1} \frac{(-1)^{i}}{2^{i}} \frac{(2 m-1) ! !}{(2 m-2 i-1) ! !} z^{2 m-2 i-1}\right. \\
\left.+\frac{(-1)^{m}}{2^{m-1}}(2 m-1) ! ! \int e^{z^{2}} d z\right),
\end{array}
$$

set

$$
H_{3}(x, y, z)=\frac{(-1)^{q}}{2^{q-1}}(2 q-1) ! ! H_{1}(x, y, z)-\frac{(-1)^{p}}{2^{p-1}}(2 p-1) ! ! H_{2}(x, y, z) .
$$

Then $H_{3}$ is also a first integral of system (14), and it is a product of $e^{z^{2}}$ by a polynomial in $x, y, z$. This shows that system (14) has a Darboux polynomial. This completes the proof of the proposition.

We remark that for polynomial differential systems in $\mathbb{R}^{n}$

$$
\begin{aligned}
& \dot{x}_{j}=k_{j} x_{j} x_{n}-x_{n}^{\ell_{j}}, \quad j=1, \ldots, n-1, \\
& \dot{x}_{n}=-1,
\end{aligned}
$$

where $k_{j}, \ell_{j}$ 's are positive integers and no less than 2 . Using similar arguments to the ones of the proof of Proposition 3 we can prove that system (16) is Liouvillian integrable, and has or may not have a Darboux polynomial for suitable choices of the values of $k_{j}$ and $\ell_{j}$. But we need to discuss more cases, so we omit it.

Proof of Proposition 4. Consider the Abel polynomial differential equation of the form

$$
\dot{x}=1, \quad \dot{y}=-2 x y^{2}+y^{3} .
$$

Clearly, this system satisfies the degree condition of the proposition and has the Darboux polynomial $F(x, y)=y$. It was proved in [3] that 
system (17) has the non-Liouvillian first integral

$$
H(x, y)=\frac{x \operatorname{Ai}\left(x^{2}-\frac{1}{y}\right)+\operatorname{Ai}\left(1, x^{2}-\frac{1}{y}\right)}{x \operatorname{Bi}\left(x^{2}-\frac{1}{y}\right)+\operatorname{Bi}\left(1, x^{2}-\frac{1}{y}\right)} .
$$

To obtain such first integral we transform the Abel polynomial differential equation (17) into system

$$
\frac{d y}{d x}=-2 x y^{2}+y^{3}
$$

Note that the birational map

$$
X=x^{2}-\frac{1}{y}, \quad Y=x,
$$

transforms system (18) into the Riccati equation $\frac{d Y}{d X}=Y^{2}-X$. In this case the Abel equation has a non-Liouvillian first integral, where the transcendental functions in the first integral are in the variable $x^{2}-\frac{1}{y}$. The change has been obtained by taking $x^{2}-\frac{1}{y}$ as a new variable.

Proof of Theorem 5. By contrary we assume that system (3) is Liouvillian integrable. Let $H(x, y)$ be a Liouvillian first integral of system (3). Take the change of variables

$$
x_{1}=x, \quad x_{2}=y+F(x), \quad F(x)=\int_{0}^{x} f(s) d s .
$$

System (3) is transformed to

$$
\dot{x}_{1}=x_{2}-F\left(x_{1}\right), \quad \dot{x}_{2}=-g\left(x_{1}\right) .
$$

Set $G\left(x_{1}, x_{2}\right)=H\left(x_{1}, x_{2}-F\left(x_{1}\right)\right.$. Then $G$ is Liouvillian and it is a first integral of system (20). Indeed, direct calculations show that

$$
\begin{aligned}
& \left(x_{2}-F\left(x_{1}\right)\right) \partial_{x_{1}} G\left(x_{1}, x_{2}\right)-g\left(x_{1}\right) \partial_{x_{2}} G\left(x_{1}, x_{2}\right) \\
& =\left(x_{2}-F\left(x_{1}\right)\right)\left(\partial_{x} H-f\left(x_{1}\right) \partial_{y} H\right)-g\left(x_{1}\right) \partial_{y} H \\
& =y \partial_{x} H-(f(x) y+g(x)) \partial_{y} H \equiv 0,
\end{aligned}
$$

where $\partial_{x}$ denotes the partial derivative with respect to $x$, and in the last equality we have used the fact that $H(x, y)$ is a first integral of system (3).

By assumption $\operatorname{deg} f>\operatorname{deg} g$, we have $\operatorname{deg} F>\operatorname{deg} g+1$. So we get from Corollary 2 that system (20) has a Darboux polynomial. Let $M\left(x_{1}, x_{2}\right)$ be a Darboux polynomial of system (20) with the associated cofactor $K\left(x_{1}, x_{2}\right)$. Set

$$
N(x, y)=M(x, y+F(x)), \quad L(x, y)=K(x, y+F(x)) .
$$


Then $N$ and $L$ are both polynomials. We claim that $N(x, y)$ is a Darboux polynomial of system (3) with the associated cofactor $L(x, y)$. Indeed, we get from the change (19) that

$$
\begin{aligned}
y \partial_{x} N & -(f(x) y+g(x)) \partial_{y} N \\
& =y\left(\partial_{x_{1}} M+f(x) \partial_{x_{2}} M\right)-(f(x) y+g(x)) \partial_{x_{2}} M \\
& =\left(x_{2}-F\left(x_{1}\right)\right) \partial_{x_{1}} M-g\left(x_{1}\right) \partial_{x_{2}} M \\
& =K\left(x_{1}, x_{2}\right) M\left(x_{1}, x_{2}\right) \\
& =L(x, y) N(x, y),
\end{aligned}
$$

where in the third equality we have used the fact that $M\left(x_{1}, x_{2}\right)$ is a Darboux polynomial of system (20) with cofactor $K\left(x_{1}, x_{2}\right)$. This proves that system (3) has the Darboux polynomial $N(x, y)$.

In addition, Odani [29] proved that if $f, g \not \equiv 0, \operatorname{deg} f \geq \operatorname{deg} g$ and $g / f \not \equiv$ constant, then system (3) has no a Darboux polynomial. Since our theorem is under the assumption of the Odani's theorem, system (3) cannot have a Darboux polynomial. This contradiction verifies that system (3) is not Liouvillian integrable. This completes the proof of the theorem.

\section{ACKNOWLEDGMENTS}

The first author is partially supported by a MINECO/FEDER grant MTM2008-03437, a CIRIT grant number 2009SGR-410, an ICREA Academia, two grants FP7-PEOPLE-2012-IRSES 316338 and 318999, and FEDER-UNAB10-4E-378. The second author is supported by Portuguese National Funds through FCT - Fundação para a Ciência e a Tecnologia within the project PTDC/MAT/117106/2010 and by CAMGSD. The third author is partially supported by NNSF of China grant number 11271252, by RFDP of Higher Education of China grant number 20110073110054, and by FP7-PEOPLE-2012-IRSES-316338 of Europe.

This work was done during the second and third authors visited Centre de Recerca Mathemàtica, Barcelona and Departament de Matemàtiques, Universitat Autònoma de Barcelona. We appreciate their support and hospitality.

\section{REFERENCES}

[1] J. Chavarriga, I.A. García, J. Llibre and H. Zoladek, Invariant algebraic curves for the cubic Liénard system with linear damping, Bull. Sci. Math. 130 (2006), 428-441.

[2] J. Chavarriga, H. Giacomini, J. Giné and J. Llibre, Darboux integrability and the inverse integrating factor, J. Differential Equations 194 (2003), 116-139. 
[3] E.S. Cheb-Terrab and A.D. Roche, Abel ODE's: equivalence and integrable classes, Comput. Phys. Commun. 130 (2000), 204-231.

[4] C. Christopher, Liouvillian first integrals of second order polynomial differential equations, Electron J. Differential Equations 1999 (1999), no. 49, 1-7.

[5] C. Christopher and C. Li, Limit Cycles of Differential Equations, Birkhäuser, Basel, 2007.

[6] C. Christopher, J. Llibre and J.V. Pereira, Multiplicity of invariant algebraic curves in polynomial vector fields, Pacific J. Math. 229 (2007), 63-117.

[7] G. Darboux, Mémoire sur les équations différentielles algébriques du premier ordre et du premier degré (Mélanges), Bull. Sci. Math. 2 (1878), 60-96; 123$144 ; 151-200$.

[8] G. Darboux, De l'emploi des solutions particulières algébriques dans l'intégration des systèmes d'équations différentielles algébriques, C. R. Math. Acad. Sci. Paris 86 (1878), 1012-1014.

[9] A. Gasull, J. Llibre and X. Zhang, One-Dimensional quaternion homogeneous polynomial differential equations, J. Math. Phys. 50 (2009), 082705, 17 pp.

[10] A. Gasull and V. Mañosa, A Darboux-type theory of integrability for discrete dynamical systems, J. Difference Equ. Appl. 8 (2002), 1171-1191.

[11] J. Giné, M. Grau and J. Llibre, On the extensions of the Darboux theory of integrability, Nonlinearity 26 (2013), 2221-2229.

[12] J. Giné, M. Grau and J. Llibre, A note on Liouvillian first integrals and invariant algebraic curves, Appl. Math. Letters 26 (2013), 285-289.

[13] J.P. Jouanolou, Equations de Pfaff algébriques, Lect. Notes in Math. 708, Springer-Verlag, New York/Berlin, 1979.

[14] C. Liu, G. Chen and J. Yang, On the hyperelliptic limit cycles of Liénard systems, Nonlinearity, 25 (2012), 1601-1611.

[15] J. Llibre, Integrability of polynomial differential systems, in Handbook of differential equations, Elsevier, Amsterdam, 2004, pp.437-532.

[16] J. Llibre and C. Pantazi, Darboux theory of integrability for a class of nonautonomous vector fields, J. Math. Phys. 50 (2009), 102705, 19 pp.

[17] J. Llibre, R. Ramírez and N. Sadovskaia, On the 16th Hilbert problem for algebraic limit cycles, J. Differential Equations 248 (2010), 1401-1409.

[18] J. Llibre, R. Ramírez and N. Sadovskaia, On the 16th Hilbert problem for limit cycles on nonsingular algebraic curves, J. Differential Equations 250 (2011), 983-999.

[19] J. Llibre and C. Valls, Liouvillian first integrals for Liénard polynomial differential systems, Proc. Amer. Math. Soc. 138 (2010), 3229-3239.

[20] J. Llibre and C. Valls, Liouvillian first integrals for generalized Liénard polynomial differential systems, Adv. Nonlinear Stud. 13 (2013), 825-835.

[21] J. Llibre and C. Valls, The generalized Liénard polynomial differential systems $x^{\prime}=y, y^{\prime}=-g(x)-f(x) y$ with $\operatorname{deg} g=\operatorname{deg} f+1$ are not Liouvillian integrable, preprint.

[22] J. Llibre, S. Walcher and X. Zhang, Local Darboux first integrals of analytic differential systems, Bull. Sci. Math. 138 (2014), 71-88.

[23] J. Llibre and X. Zhang, Invariant algebraic surfaces of the Lorenz systems, J. Math. Phys. 43 (2002), 1622-1645.

[24] J. Llibre and X. Zhang, On the algebraic limit cycles of Liénard systems, Nonlinearity 21 (2008), 2011-2022. 
[25] J. Llibre and X. Zhang, Darboux Theory of Integrability in $\mathbb{C}^{n}$ taking into account the multiplicity, J. Differential Equations 246 (2009), 541-551.

[26] J. Llibre and X. Zhang, Darboux theory of integrability for polynomial vector fields in $\mathbb{C}^{n}$ taking into account the multiplicity at infinity, Bull. Sci. Math. 133 (2009), 765-778.

[27] J. Llibre and X. Zhang, Rational first integrals in the Darboux theory of integrability in $\mathbb{C}^{n}$, Bull. Sci. Math. 134 (2010), 189-195.

[28] J. Llibre and X. Zhang, On the Darboux integrability of polynomial differential systems, Qual. Theory Dyn. Syst. 11 (2012), 129-144.

[29] K. Odani, The limit cycle of the van der Pol equation is not algebraic, J. Differential Equations 115 (1995), 146-152.

[30] M.F. Singer, Liouvillian first integrals of differential equations, Trans. Amer. Math. Soc. 333 (1992), 673-688.

[31] D. Schlomiuk, Algebraic particular integrals, integrability and the problem of the center, Trans. Amer. Math. Soc. 338 (1993), 799-841.

[32] S.P. Swinnerton-Dyer, The invariant algebraic surfaces of the Lorenz system, Math. Proc. Camb. Phil. Soc. 132, 385-393.

[33] C. Valls, Invariant algebraic surfaces for generalized Raychaudhuri equations, Comm. Math. Phys. 308 (2011), 133-146.

[34] X. Yu and X. Zhang, The hyperelliptic limit cycles of the Liénard systems, J. Math. Anal. Appl. 376 (2011), 535-539.

[35] X. Zhang, Invariant algebraic curves and rational first integrals of holomorphic foliations in $\mathbb{C} P(2)$, Science in China Ser. A 46 (2003), 271-279.

[36] X. Zhang, Global structure of quaternion polynomial differential equations, Comm. Math. Phys. 303 (2011), 301-316.

[37] X. Zhang, The 16th Hilbert problem on algebraic limit cycles, J. Differential Equations 251 (2011), 1778-1789.

[38] X. Zhang, Liouvillian integrability of polynomial differential systems, Trans. Amer. Math. Soc., to appear.

[39] X. Zhang, Integrability of Dynamical Systems: Algebra and Analysis, preprint, 2014.

[40] H. Zoladek, Algebraic invariant curves for Liénard equation, Trans. Amer. Math. Soc. 350 (1998), 1681-1701.

${ }^{1}$ Departament de Matemàtiques, Universitat Autònoma de Barcelona, 08193 Bellaterra, Barcelona, Catalonia, Spain

E-mail address: jllibre@mat.uab.cat

2 Departamento de Matemática, Instituto Superior Técnico, Universidade Técnica de Lisboa, Av. Rovisco Pais 1049-001, Lisboa, PorTUGAL

E-mail address: cvalls@math.ist.utl.pt

3 Department of Mathematics, MOE-LSC, Shanghai Jiao tong UniVersity, Shanghai, 200240, P. R. China

E-mail address: xzhang@sjtu.edu.cn 